\title{
Isolated Pancreatic Transection Due to Motorcycle Accident with Endoscopic Treatment: A Case Report
}

\author{
Chan Ik Park, M.D.*, Sung Jin Park, M.D.*, Jae Hun Kim, M.D.*, Dong Hoon Baek, M.D. ${ }^{\dagger}$ \\ *Department of Trauma Surgery, ${ }^{\dagger}$ Division of Gastroenterology, Department of Internal Medicine, \\ Biomedical Research Institute, Pusan National University Hospital, Busan, Korea
}

Correspondence to:
Sung Jin Park, M.D.
Department of Trauma Surgery,
Pusan National University
Hospital, 179 Gudeok-ro,
Seo-gu, Busan 49241, Korea
Tel: +82-51-240-7369
Fax: +82-51-247-7719
E-mail: psjtrauma@gmail.com
ORCID:
http://orcid.org/0000-0002-4852-5442

\begin{abstract}
An isolated pancreatic transection due to blunt trauma is a rare occurrence that usually requires surgical treatment. Non-surgical treatment for a pancreatic transection remains controversial because of its associated complications. On the other hand, non-surgical treatment has been used increasingly as a treatment option with promising results in recent years. A patient presented with a suspected pancreatic injury caused by a motorcycle accident. The computed tomography findings revealed an isolated pancreatic neck transection with a small amount of fluid collection. He was hemodynamically stable without signs of peritoneal irritation. Endoscopic retrograde pancreatography and stent insertion were performed. The patient had no significant complications and was discharged on day 18. The stent was removed on day 103 and the patient showed good recovery. For an isolated pancreatic transection, endoscopic intervention can be considered as an alternative with a good outcome in selected patients. (J Acute Care Surg 2018;8:74-77)
\end{abstract}

Key Words: Cholangiopancreatography, endoscopic retrograde, Pancreas, Wounds and Injuries

Received March 22, 2018, Revised August 27, 2018, Accepted September 3, 2018

Copyright $(2018$ by Korean Society of Acute Care Surgery

(c) This is an Open Access article distributed under the terms of the Creative Commons Attribution Non-Commercial License (http://creativecommons.org/licenses/by-nc/4.0) which permits unrestricted non-commercial use, distribution, and reproduction in any medium, provided the original work is properly cited.

ISSN 2288-5862(Print), ISSN 2288-9582(Online)

https://doi.org/10.17479/jacs.2018.8.2.74

\section{Introduction}

Pancreatic injury caused by blunt trauma is rare, and isolated pancreatic injury is rarer [1]. Such injuries result in high morbidity and mortality rates usually due to delayed diagnosis and treatment [2]. Early diagnosis of pancreatic injury is challenging because of its presentation and subtle symptoms due to the retroperitoneal location of this organ. Generally, low-grade pancreatic injury can be nonoperatively managed. However, operative management is recommended for high-grade injuries, involving main pancreatic duct (MPD) [3]. Certain studies have demonstrated increased morbidity and mortality because of delayed surgery in patients with high-grade pancreatic injuries who initially nonoperatively treated [4]. However, due to the development of intervention skills and intensive care, nonoperative management, including endoscopic and radiologic intervention has become a promising treatment option in recent years [5].

We report a case of isolated transection of the pancreatic neck after blunt trauma that was successfully managed with endoscopic stenting.

\section{Case Report}

A 16-year-old boy was transferred to the regional trauma center of Pusan National University Hospital after a motor vehicle accident. He complained of mild abdominal pain and focal 
tenderness in the epigastric area. Initial vital signs were stable; blood pressure 120/80 $\mathrm{mmHg}$, heart rate 106/min, and respiratory rate $16 / \mathrm{min}$. Laboratory findings were significant for white blood count $20.49 / \mu 1$ (reference range, 4,000 11,000/ $\mu 1$ ), mildly elevated amylase $102 \mathrm{IU} / \mathrm{L}$ (reference range, 36 128 IU/L), and lipase $220 \mathrm{U} / \mathrm{L}$ (reference range, $22 \sim 51 \mathrm{U} / \mathrm{L}$ ) and other values within normal range.

Abdominal contrast enhanced computed tomography (CT) revealed transection at the neck of the pancreas with a small peripancreatic fluid collection (Fig. 1). No evidence of other intra-abdominal organ injury was found. Magnetic resonance cholangiopancreatography (MRCP) of the pancreatic duct was indicative MPD injury (Fig. 2). His vital signs were stable, abdominal pain was less than moderate, and he had no other intra-abdominal organ injury. Therefore we decided on nonoperative management and the patient was admitted to the trauma intensive care unit. Surgical treatment was also planned if the patient did not receive endoscopic retrograde cholangiopancreatography (ERCP) within 24 hours, the procedure was failed or the patient's condition was aggravated.

The next day, ERCP, performed within 24 hours after injury for evaluation and nonoperative management of the MPD injury, demonstrated complete disruption of the MPD on the neck (Fig. 3), which is a grade III injury according to the American Association for the Surgery of Trauma. A 5 French stent was placed to cross the injured site. On day 3, follow-up CT scan demonstrated no significant interval change. After intensive care of four days, the patient was transferred to the general ward for conservative care. On day 10, follow-up CT scan showed no interval change, and enteral feeding was started. On day 17, follow-up CT was similar as before, and he was discharged without any significant complication 18 days after the trauma.

On day 33, CT scan revealed reduction of the peripancreatic fluid collection. On day 34, a second ERCP demonstrated contrast leakage from the injured site of the pancreatic duct, so the stent was changed. The patient remained stable and did not show any other symptom. Lastly, the third ERCP on day 99 revealed no contrast leakage. The fluid collection also was reduced on follow-up CT scan. After four days, on day 103, we successfully removed the stent. The patient was followed-up in the outpatient clinic without any significant complication.

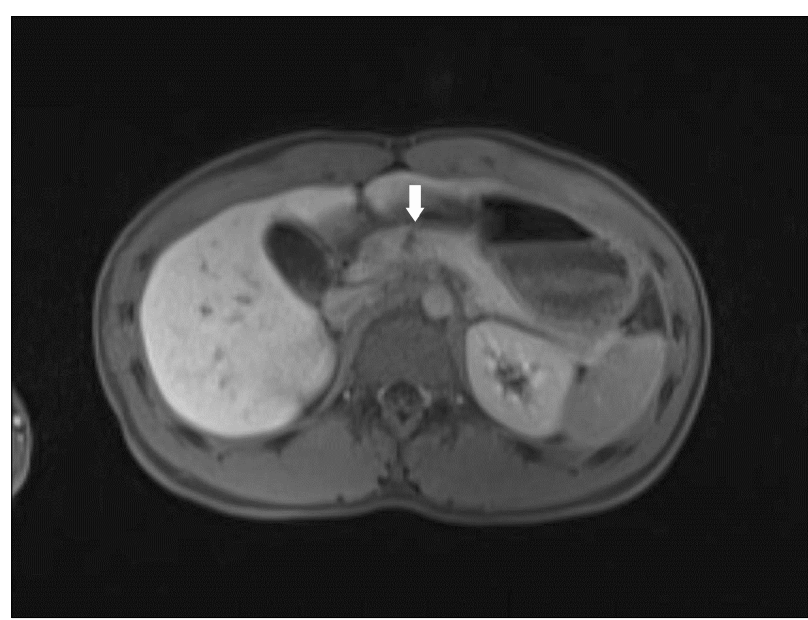

Fig. 2. Magnetic resonance cholangiopancreatography showing transection at the pancreas neck (arrow).
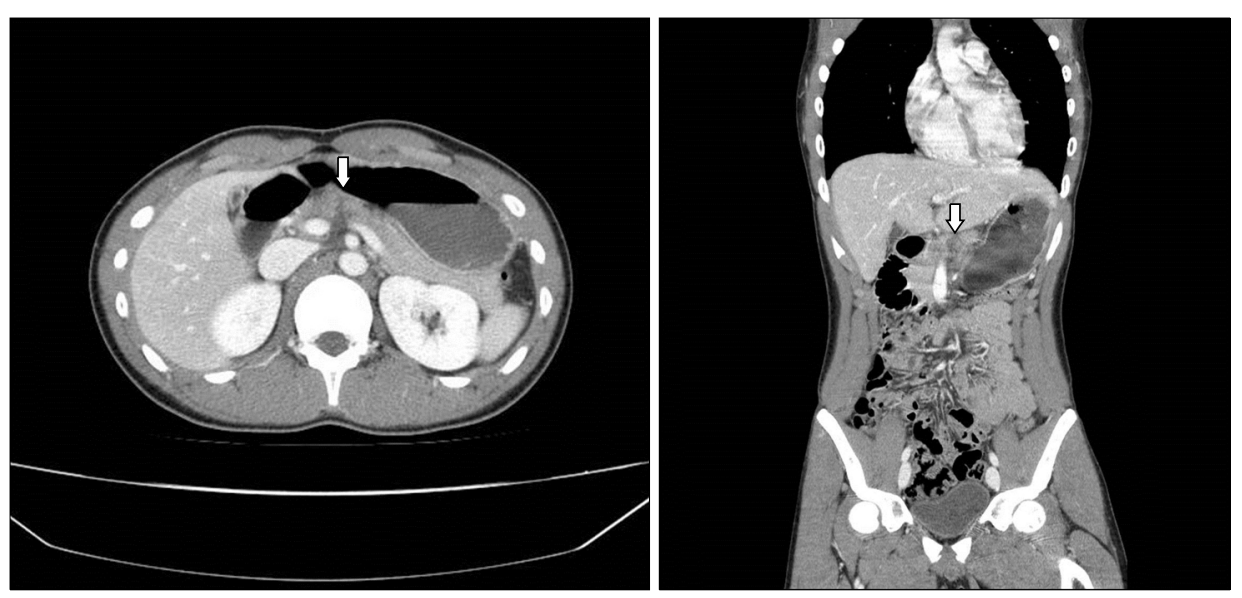

Fig. 1. Computed tomography of abdomen suggesting transection at the neck of the pancreas with small amount of peripancreatic fluid collection (arrows). 

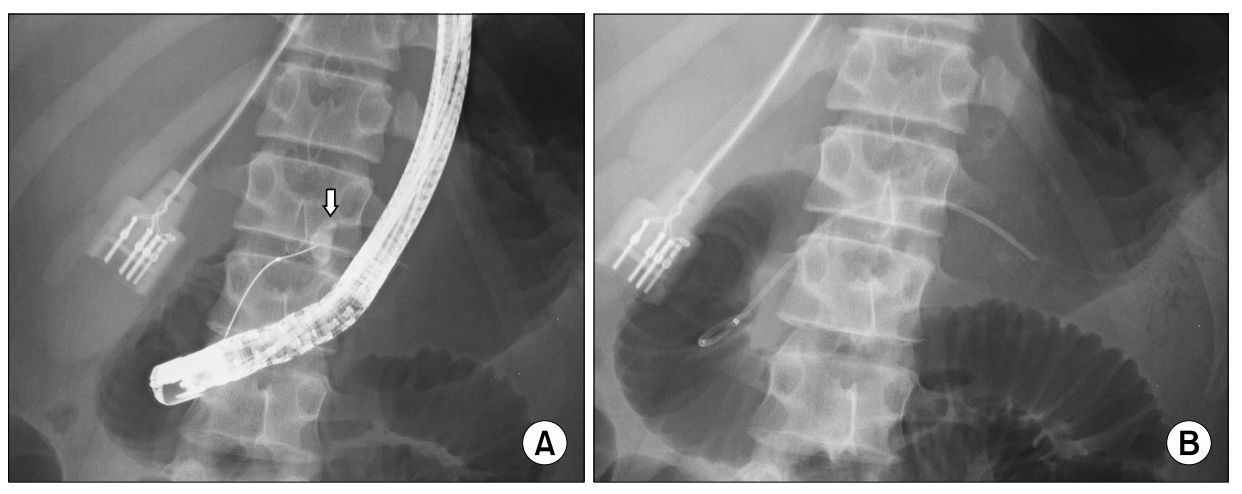

Fig. 3. (A) Endoscopic retrograde cholagiopancreatography demonstrating pancreatic duct complete injury with contrast leakage (arrow). (B) A pancreatic stent was placed to cross the injured site.

\section{Discussion}

Pancreatic injury is rare, occurring in 3.1\% of blunt abdominal traumas, and isolated pancreatic injury is rarer $(0.7 \%$ of blunt abdominal traumas), but they are associated with morbidity and a mortality rate s of up to $12 \%$ and $34 \%$ respectively $[1,2]$. Because of the proximity of adjacent solid and hollow organs and vascular structures, associated injuries are common [6]. Due to the retroperitoneal location, clinical signs and symptoms are initially vague and subtle. In addition, there is no laboratory finding that is specific for pancreatic injury. Therefore, delayed diagnosis is common and leads to high morbidity and mortality rates, especially in patients with MPD injury. Early diagnosis of MPD injury is most crucial after blunt pancreatic trauma.

For blunt pancreatic injury, abdominal CT scan (contrast enhanced) is the first choice of image study. However, during the early period after trauma, the sensitivity and specificity of CT scans for MPD injury are not satisfactory [7]. When CT findings are inconclusive but suspicious according to the injury mechanism, further investigation with other image studies or procedures should be conducted. Next, if the patient is hemodynamically stable, the pancreatic injury is not significant and there is no other intra-abdominal organ injury, MRCP or ERCP can be performed. MRCP allows for noninvasive evaluation of not only the integrity of the pancreatic duct but also pancreatic parenchyma and the surrounding tissues [7]. Despite its own complications, we prefer ERCP because it can be performed with the possible stenting of the MPD. We could find the pancreatic injury on the initial CT scan, but it was necessary to identify the presence of main duct injury. If there is a main duct injury, operative treatment or endoscopic intervention should be performed within 24 hours to achieve good results. Once the patient was young and the symptoms were mild and there was a small amount of fluid collection around the pancreas, we considered non-operative treatment first. However, there are not many hospitals where ERCP is available 24 hours a day, 365 days a year. In our institution, one endoscopic interventionist is in charge of the entire hospital. Therefore, generally, ERCP Can be performed only during daytime on a specific day of the week (monday, wednesday, and thursday), and the procedure was not performed within 24 hours as routine. After identifying the main duct injury with MRCP, we consulted with the interventionist and ERCP and pancreatic stent insertion was able to be performed emergently on the day when the procedure was not possible routinely.

ERCP is considered the golden standard for the diagnosis of the pancreatic duct injury. ERCP reveals the integrity of the pancreatic duct and the injured site and extent; thus decisions for the appropriate method of management are possible. According to the condition of the injury, endoscopic treatments, such as stenting to bridge the injured site and sphincterotomy can be simultaneously performed.

Controversies regarding the operative or nonoperative treatment of grade III or IV pancreatic injury exist. Regardless of the grade III or IV injury, the primary challenge is restrict MPD leakage. In their guideline for management of adult pancreatic injuries, the Eastern Association for the Surgery of Trauma recommends operative management for grade III or IV pancreatic injury. They stated that treatment delays likely contribute to morbidity and mortality due to regularly occurring treatment 
failure after nonoperative treatment [3]. However, due to the development of intervention skills and intensive care, successful nonoperative management for pancreatic injury have increased.

Bhasin et al. [4] recommend that patients with duct distuption, especially partial, that can be bridged should undergo transpapillary pancreatic duct stenting. However, patients with complete duct disruption should be surgically treated. Recently, several cases of successful treatment of grade III pancreatic injury with endoscopic management have been reported [8]. Further studies are required for confirming whether nonoperative management improves morbidity and mortality.

Lin et al. [9] reported the long-term results of endoscopic stenting for blunt major pancreatic duct injury. In their study, three of six patients had severe MPD stricture as a complication of stenting or the injury itself and the stent was maintained for a long duration (26 43 months). Our patient was asymptomatic for the seven months of follow-up and needs long-term observation.

Other concerns regarding nonoperative management of pancreatic injury include post-ERCP pancreatitis and the requirement of additional intervention. Presently, the definition of post-ERCP pancreatitis is widely accepted fulfillment of two or more of the above three criteria: a serum amylase and or lipase level at least three times the upper limit of normal range in the presence of abdominal pain and/or radiologic findings of inflammation of the pancreas [10]. In the present case, the pancreatic enzyme level was elevated after ERCP but the patient did not complain of abdominal pain. After few days, the elevated pancreatic enzyme normalized. Multiple ERCPs and endoscopy for stent removal could potentially harm the patient. Our patient underwent three ERCPs and finally endoscopy for stent removal without significant complications. However, the patient avoided laparotomy under general anesthesia and complications of pancreatic resection.

Isolated pancreatic transection is rare and is traditionally managed by laparotomy. However, we successfully treated an MPD injury with ERCP and stenting. In selected patients, endoscopic treatment can be an attractive therapeutic option, avoiding the requirement for laparotomy.

\section{Acknowledgments}

This work was supported by clinical research grant from Pusan National University Hospital in 2017.

\section{Conflicts of Interest}

No potential conflict of interest relevant to this article was reported.

\section{References}

1. Siboni S, Kwon E, Benjamin E, Inaba K, Demetriades D. Isolated blunt pancreatic trauma: a benign injury? J Trauma Acute Care Surg 2016;81:855-9.

2. Kao LS, Bulger EM, Parks DL, Byrd GF, Jurkovich GJ. Predictors of morbidity after traumatic pancreatic injury. J Trauma 2003;55:898-905.

3. Ho VP, Patel NJ, Bokhari F, Madbak FG, Hambley JE, Yon $\mathrm{JR}$, et al. Management of adult pancreatic injuries: a practice management guideline from the Eastern Association for the Surgery of Trauma. J Trauma Acute Care Surg 2017;82: 185-99.

4. Bhasin DK, Rana SS, Rao C, Gupta R, Verma GR, Kang M, et al. Endoscopic management of pancreatic injury due to abdominal trauma. JOP 2012;13:187-92.

5. Björnsson B, Kullman E, Gasslander T, Sandström P. Early endoscopic treatment of blunt traumatic pancreatic injury. Scand J Gastroenterol 2015;50:1435-43.

6. Krige JE, Beningfield SJ, Nicol AJ, Navsaria P. The management of complex pancreatic injuries. S Afr J Surg 2005;43: 92-102.

7. Rekhi S, Anderson SW, Rhea JT, Soto JA. Imaging of blunt pancreatic trauma. Emerg Radiol 2010;17:13-9.

8. Vezakis A, Koutoulidis V, Fragulidis G, Polymeneas G, Polydorou A. Complete traumatic main pancreatic duct disruption treated endoscopically: a case report. J Med Case Rep 2014;8:173.

9. Lin BC, Liu NJ, Fang JF, Kao YC. Long-term results of endoscopic stent in the management of blunt major pancreatic duct injury. Surg Endosc 2006;20:1551-5.

10. Guda NM, Reddy DN, Kumar A. Complications of ERCP. Indian J Gastroenterol 2014;33:1-9. 